\title{
FROBENIUS AND THE HODGE FILTRATION
}

\author{
BY B. MAZUR
}

The problem of $p$-adically estimating the number of solutions of an algebraic variety over a finite field of characteristic $p$ was initiated by the classical result of Chevalley-Warning [11].

THEOREM. Let $G\left(x_{1}, \ldots, x_{n}\right)$ be a polynomial with integral coefficients, of degree less than $n$. The number of solutions of

$$
G\left(x_{1}, \ldots, x_{n}\right) \equiv 0 \bmod p
$$

is divisible by $p$.

My aim in this paper, is to explain a conjecture of N. Katz [3], which provides quite sharp information concerning this general problem, and to indicate some theorems I have obtained which affirm his conjecture, under a mild hypothesis. Proofs of these theorems will be contained in [8].

1. The Zeta-function of a variety over a finite field. Let $X_{0}$ be a scheme of finite type over $k=F_{q}$. We wish to study $N_{s}$, the number of rational points of $X_{0}$ over $F_{q^{s}}$, for all $s>1$. The Zeta-function of $X_{0} / k$ expresses this information for us:

$$
Z\left(T ; X_{0} / k\right)=\exp \left(\sum_{s=1}^{\infty} \frac{N_{s} T^{s}}{s}\right) .
$$

Given any "Weil cohomology" in the terminology of Kleiman's survey article [6], the Zeta-function may be expressed as an alternating product of characteristic polynomials,

$$
Z\left(T ; X_{0} / k\right)=\prod_{i} \operatorname{det}\left(1-f_{i} T\right)^{(-1)^{i+1}}
$$

where $f_{i}$ denotes the endomorphism of $H^{i}\left(X_{0}\right)$ induced by $f$, the $q$ th power endomorphism of the structure sheaf of $X_{0}$. Moreover, if $X_{0}$ is proper and smooth, the Zeta-function satisfies a functional equation with respect to a change of variables

An expanded version of an invited address delivered to the 688th meeting of the Society at the Massachusetts Institute of Technology in Cambridge, Massachusetts on October 30, 1971; received by the editors January 18, 1972.

AMS 1970 subject classifications. Primary 14F30, 14G15, 14G20; Secondary 14G10.

Key words and phrases. Canonical lifting of Frobenius on de Rham cohomology, Newton polygon, crystal, span. 


$$
T \rightarrow q^{d} / T
$$

such that the $i$ th factor in the above decomposition is sent to the $(2 d-i)$ th.

The above decomposition expresses the Zeta-function as an alternating product of polynomials whose coefficients lie in the field of coefficients of the particular Weil cohomology that we are considering.

It is conjectured (loc. cit.) that the above decomposition,

$$
Z\left(T ; X_{0} / k\right)=\prod_{i} P_{i}(T)^{(-1)^{l+1}},
$$

has the property that the $P_{i}$ are polynomials with integral coefficients, and they are independent of which Weil cohomology theory one takes.

This is indeed the case for curves, abelian varieties, surfaces, and smooth complete intersections in projective space.

Let us settle on a smooth proper $X_{0}$ for the remainder of our discussion. Also, for this paragraph, let us speak as if the above conjecture were true. Then if $\alpha$ is the reciprocal of a root of one of the $P_{i}, \alpha$ is an algebraic integer. Moreover, thanks to the functional equation, so is $q^{d} / \alpha$. We may conclude that $\alpha$ is an $l$-adic unit, for any $l \neq p$. There remain two questions of an adelic nature concerning $\alpha$.

(I) "The Riemann Hypothesis:" What are the complex absolute values of $\alpha$ and its conjugates?

(II) What are the $p$-adic absolute values of $\alpha$ and its conjugates?

Of course, it is expected that the answer to question (I) lies far deeper than an answer to question (II).

Whereas an understanding of question (I) would lead to (the wellknown) inequalities bounding the number of rational points on $X_{0}$ over $k$, a determination of (II) would have as a consequence information concerning the number of rational points modulo powers of $p$ over $k$ and its extension fields.

Question (II) has been pursued most vigorously by Dwork, who has fashioned a magnificent theory designed to come to an understanding of this question in great depth, especially for hypersurfaces.

I found that a good introduction, for the nonexpert, to the $p$-adic theory of Dwork is to read a beautiful paper of Manin, where the $\bmod p$ theory for curves is treated (On the Hasse-Witt matrix of an algebraic curve [7]) and then to read Dwork's ( $A$ deformation theory for the zeta-function of a hypersurface [2]) and Katz's (On the differential equations satisfied by period matrices [4]).

It may also be enlightening to review the classical theorem of Stickelberger concerning the $p$-adic ordinal of Gauss and Jacobi sums, attempting to view it as fitting into the tradition of question (II) [13]. 
It should be evident that if one is to pursue question (II) by a cohomological theory-by constructing, perhaps, a Weil cohomology-the coefficient field should be either the $p$-adic numbers, or an extension field, so that one may make $p$-adic estimates naturally.

The first such theory (aside from Dwork's own) was due to MonskyWashnitzer, and more recently there have been others: one developed by Lubkin, and another, crystalline cohomology, initiated by Grothendieck, and developed to a very large extent by Berthelot [1]; for the definitive version of this theory, one awaits the publication of his thesis.

Here is a modest consequence of the recent work of Berthelot:

Let $X / W$ be proper and smooth, where $W=W(k)$ is the Witt vectors of the finite field $k$. Let $X_{0} / k$ denote the closed fibre. Then the de Rham cohomology groups $H_{D R}(X / W)$ depend only on $X_{0}$ and not on the particular lifting $X$ of $X_{0}$ to $W$. The Frobenius endomorphism $F$ of $X_{0}$ may very well not lift to an endomorphism of $X$ (indeed, it rarely does); nevertheless, it is a consequence of the crystalline theory that one may define a canonical "lifting of Frobenius" $F: H_{D R}(X / W) \rightarrow H_{D R}(X / W)$ which is functorial in $X_{0} / k$.

Moreover, there is a decomposition of the Zeta-function,

$$
Z\left(T ; X_{0} / k\right)=\prod_{i} \operatorname{det}\left(1-f_{i}^{(a)} T\right)^{(-1)^{i+1}}
$$

where $f_{i}^{(a)}$ is the endomorphism of $H_{D R(X / W)}^{i}$ induced by the $a$ th power of the 'canonical lifting of Frobenius', where $q=p^{a}$.

It is this theory of Berthelot that we wish to use to examine question (II), which we now paraphrase:

QUeSTION. Let $\boldsymbol{F}$ denote the 'canonical lifting of Frobenius' operating on $H_{D R}^{m}(X / W)$. If $K$ is the field of fractions of $W$, consider the semilinear endomorphism of the $K$-vector space $H_{D R}^{m}(X / W) \otimes K$ induced by $F$. Then $F^{a}$ is $W$-linear, and we may pass to a finite field extension $L / K$ which contains the eigenvalues of $F^{a}$. If ord denotes the valuation on $L$ such that $\operatorname{ord}(q)=1$, what can we say about the values $\operatorname{ord}(\alpha)$ for the eigenvalues $\alpha$ of $F^{a}$ ?

There is a beautiful conjecture of Katz which links the values ord $(\alpha)$ by a series of inequalities to the hodge numbers of $X$. This conjecture is most elegantly described by means of 'Newton polygons'; but by way of introduction, it will do little harm to begin by bluntly listing the inequalities:

Let the "Hodge numbers" $h^{i}=h^{i, m-i}$ denote the dimension of the vector space $H^{m-i}\left(X_{K}, \Omega_{X / K}^{i}\right)$. Set $\beta^{i}=h^{0}+h^{1}+\ldots+h^{i}$. Let $\alpha_{i}$ denote the eigenvalues of $F^{a}$ on $H_{D R}^{m}(X / W)$, and arrange them so that $a_{i}=$ ord $\alpha_{i}$ are nondecreasing. Then, 


$$
0 \leqq a_{1} \leqq a_{2} \leqq \ldots \leqq a_{\beta} \leqq m, \quad \text { where } \beta=\operatorname{dim} H_{D R}^{m}\left(X_{K} / K\right) .
$$

FIRST FORM OF THE CONJECTURE. Let $t$ be any positive integer less than or equal to $\beta$. Write $\beta^{j}<t<\beta^{j+1}$ and set $r=t-\beta^{j}$. Then

$$
a_{1}+\ldots+a_{t} \geqq 0 \cdot h^{0}+1 \cdot h^{1}+\ldots+j \cdot h^{j}+(j+1) \cdot r .
$$

To get a sense of what the above conjecture says, here are some consequences: There are at most $h^{0}$ eigenvalues $\alpha_{i}$ which are $p$-adic units. If this maximum is attained, that is, if there are $h^{0}$ eigenvalues which are $p$-adic units, then all the remaining eigenvalues are divisible by $q$ and there are at most $h^{1}$ of those, such that $\alpha_{i} / q$ are $p$-adic units-and so on. An application is the following important

DIVISIBILITY COROLLARY. Let $C$ be the smallest integer such that $h^{c}$ is nonzero. Then all eigenvalues $\alpha_{i}$ are divisible by $q^{c}$.

The above conjecture should be strengthened in various ways. First, giving a suitable interpretation to the word "eigenvalue" we may deal directly with the semilinear endomorphism $\boldsymbol{F}$, and then we shall be able to pose the conjecture for any perfect field - not necessarily finite. Secondly, we may deal with nonliftable varieties $X_{0} / k$ as follows:

Define the reduced Hodge numbers $\hbar^{i, m-i}$ of $X_{0}$ by

$$
\hbar^{i, m-i}=\operatorname{dim}_{k}\left(E_{\infty}^{i, m-i}\right)
$$

where the term on the right is the $E_{\infty}^{i, m-i}$ term of the Hodge spectral sequence,

$$
E_{1}^{i, m-i}=H^{m-i}\left(X_{0}, \Omega_{X_{0}}^{i}\right) \Rightarrow H_{D R}^{m}\left(X_{0} / k\right) .
$$

Define the $\bmod p$ Hodge polygon of $X_{0}$ to be the polygon constructed by means of the reduced Hodge numbers

$$
\bar{h}^{0, m}, h^{1, m-i}, \ldots, \bar{h}^{m, 0} .
$$

CONJECTURE FOR NONLIFTABLE VARIETIES. The eigenvalues of $\boldsymbol{F}^{a}$ operating on the crystalline cohomology group $H_{\mathrm{cris}}^{m}\left(X_{0} W\right)$ submit to the above system of inequalities, where one takes the reduced Hodge numbers in place of the $h^{i}$.

It is a theorem of Dwork that the above system of inequalities holds for hypersurfaces.

(See [2] where this is proved for hypersurfaces of degree prime to $p$.)

The "Divisibility Corollary" was established by Katz for smooth complete intersections in projective space [3]. 
The reader may convince himself that the functional equation alone is all that is needed to establish the above system of inequalities for curves, or abelian varieties.

2. Newton polygons. Let us begin with a well-known proposition in semilinear algebra.

Proposition (Dieudonné, MANin). Let $k$ be algebraically closed of characteristic $p$. Let $K$ be the field of fractions of $W(k)$. Let $V$ be a finitedimensional vector space over $K$ admitting a Frobenius-linear automorphism $T$, which preserves $a W(k)$-lattice in $V$.

Thus, $V$ may be regarded as a module over the noncommutative ring $A=K[T]$, of polynomials in $T$ with coefficients in $K$ such that

$$
\boldsymbol{F}(x) \cdot T=T \cdot x
$$

for $x \in K$, where $\boldsymbol{F}$ is Frobenius.

Then $V$ may be expressed uniquely as a direct sum of A-modules

$$
V=\bigoplus_{i=1}^{i=t} V_{r_{i}, s_{i}} \quad \text { where } r_{i} / s_{i}<r_{i+1} / s_{i+1}
$$

and $V_{r, s}$ is the A-module $A / A\left(T^{s}-p^{r}\right), r \geqq 0, s \geqq 1$, both integers.

The quantities $r_{i} / s_{i}$ are called the slopes of $V$, and one says that the slope $r_{i} / s_{i}$ occurs in $V$ with multiplicity $s_{i}$. Thus, a knowledge of the slopes of $V$ and their multiplicities is equivalent to a knowledge of $V$, up to isomorphism.

If $k$ is a finite field of $q=p^{a}$ elements, and $M$ a module of finite type over $W(k)$ possessing an injective Frobenius-linear endomorphism $T$, then the quantities $\operatorname{ord}_{q}(\alpha)$ where $\alpha$ ranges through the eigenvalues of $T^{a}$ are precisely the slopes (counting multiplicities) of the $A$-module $V=M \otimes_{W(k)} K$, where $K$ is the field of fractions of $W(\bar{k})$.

Consider the set of quantities,

$$
\left(r_{1}, s_{1}\right),\left(r_{2}, s_{2}\right), \ldots,\left(r_{t}, s_{t}\right)
$$

and define the Newton polygon of $\left(^{*}\right)$ to be the convex polygon (= graph of a convex piecewise linear function) in the plane, whose left-most point is the origin $(0,0)$ and which has slope $r_{i} / s_{i}$ over the interval of the horizontal axis $\left[s_{1}+\ldots+s_{i-1}, s_{1}+\ldots+s_{i}\right]$. Its right-most point lies above the point $s_{1}+\ldots+s_{t}$ on the horizontal axis.

Thus the Newton polygon of $V$ (which is, by definition, the Newton polygon of its slopes and multiplicities as described by $(*))$ completely determines $V$, up to isomorphism. 


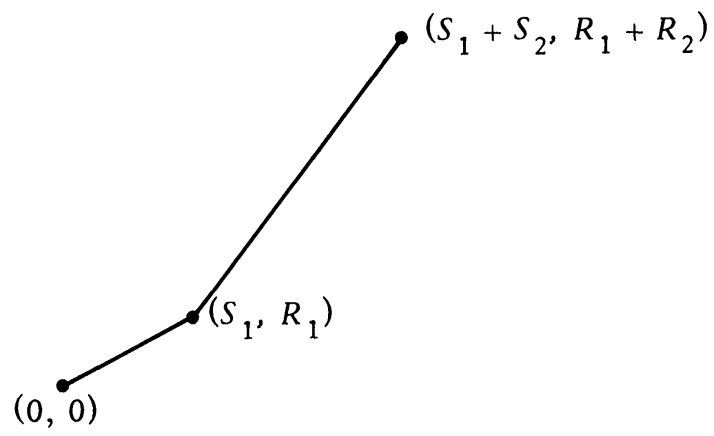

FIGURE 1

Return to a smooth proper scheme $X / W$, where $W$ is the Witt vectors of any perfect field of characteristic $p$. By the m-dimensional Newton polygon of $X / W$, we shall mean the Newton polygon of $H^{m}(X / W) \otimes_{W} K$, endowed with the 'canonical lifting of Frobenius,' where $K$ is the field of fractions of $W(k)$.

By the m-dimensional Hodge polygon of $X / W$ we shall mean the convex polygon in the plane whose left-most point is the origin and which has slope $j$ over the interval of the horizontal axis

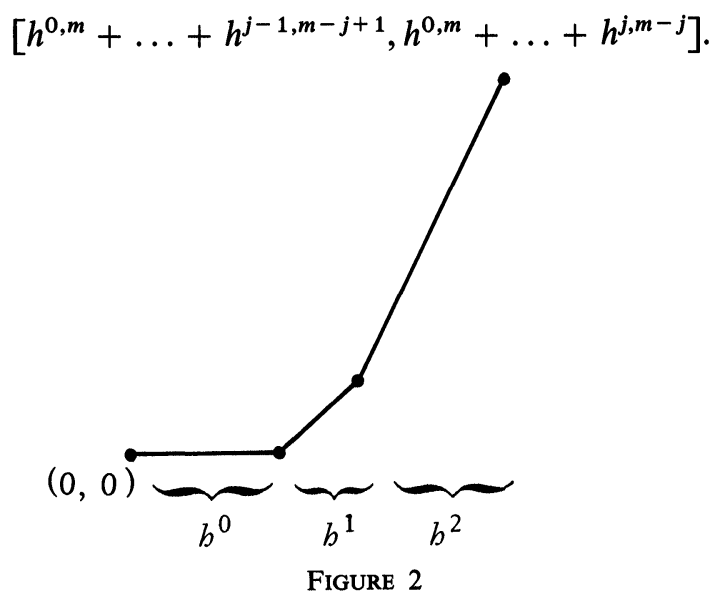

Now, Katz's conjecture may be paraphrased:

SECOND FORM OF THE CONJECTURE. The (m-dimensional) Newton polygon of $X / W$ lies above (or on) the (m-dimensional) Hodge polygon of $X / W$.

The Newton polygon is a very convenient geometric way of expressing the system of inequalities given in $\S 1$. For example, (a) the break-points of the Newton polygon must occur at integral lattice points; (b) Poincaré 


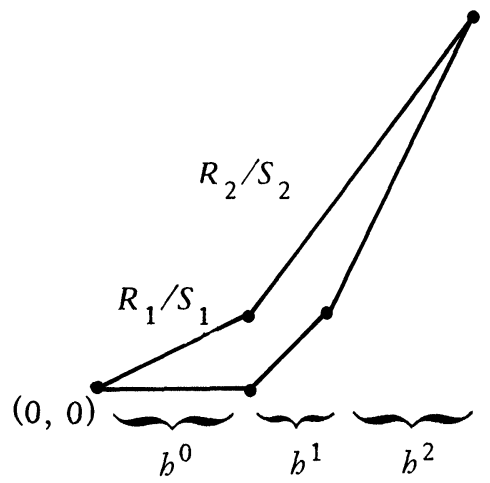

FiguRe 3

duality and, if $X / W$ is a projective variety, the strong Leftchetz theorem give us that the right-most part of the Newton and Hodge polygons coincide: it is the point $(\beta, \beta m / 2)$.

EXAMPLES. 1. $X=a$ curve of genus 3 . The five different Newton polygons (for one-dimensional cohomology) allowed by the restraint of Poincare duality are drawn in the diagram below. Note that the conjecture of Katz introduces no new restraints for a curve. Do all five possible configurations occur as actual Newton polygons of curves?

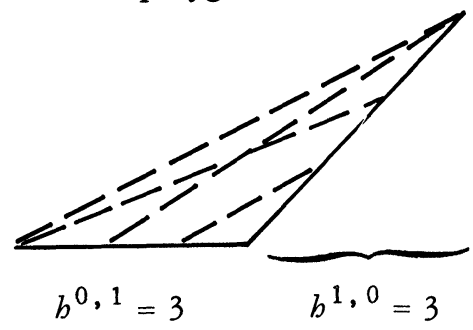

FIGURE 4

2. $X=a$ smooth proper surface such that $h^{0,2}=1$. Then the possible configurations for two-dimensional Newton polygons are quite restricted by the conjecture of Katz. (See Figure 5.)

A polygon fulfilling the conditions imposed upon it by

(a) the conjecture of Katz,

(b) Poincaré duality,

(c) the fact that there must exist at least one algebraic cycle on the surface,

can be described totally by stipulating an integer $h$ such that $0 \leqq 2 h<b_{2}$.

The polygon associated to the integer $h$ is the one possessing slopes $1-1 / h, 1,1+1 / h$ with multiplicities $h, b_{2}-2 h, h$ respectively. (See Figure 5.) 


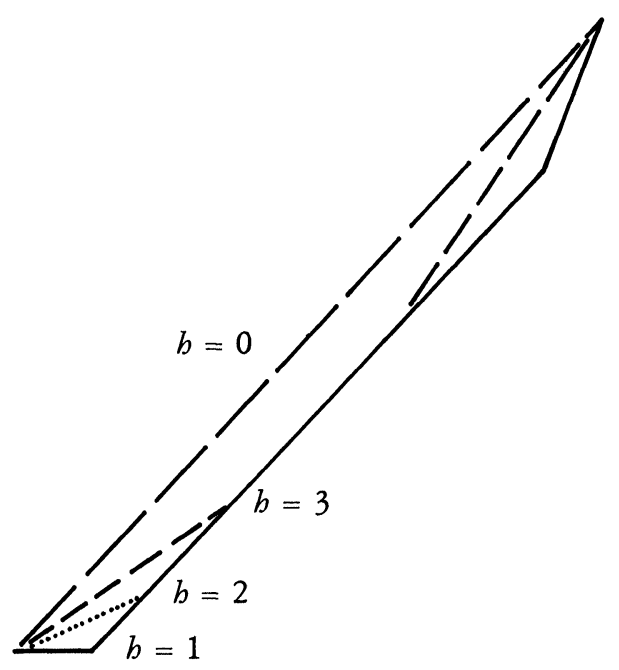

FIGURE 5

My personal interest in the p-adic analysis of the eigenvalues of Frobenius began when I came across the invariant $h$ described above, from a fundamentally different direction. In some cases this number $h$ can be viewed as the height of a $p$-divisible formal group on one parameter that $M$. Artin and I can attach to the surface $X$.

Using the theory of Berthelot, I have recently been able to establish the validity of the above conjecture, under a mild hypothesis:

THEOREM 1. The above conjecture is correct under the assumptions that $H^{q}\left(X, \Omega_{X / W}^{p}\right)$ are torsion-free $W$-modules, and $X / W$ is a projective smooth scheme.

ReMARK. The assumption that $X / W$ is projective is certainly a capricious one. It serves the purpose of enabling one to use a version of the crystalline cohomology which has the air of being more 'familiar.'

The assumption of torsion-freeness is more serious. It is satisfied in many cases of interest (e.g., smooth complete intersections in projective space), but is undoubtedly irrelevant to the final result, provided that one is willing to work under the weight of the machinery of derived categories. This looks interesting and may shed light on the relationship between the nondegeneration of the $\bmod p$ Hodge spectral sequence, and the existence of torsion in crystalline cohomology.

3. Ordinary varieties, specialization and further examples. Let us give ourselves some exceptionally well-behaved family $\left\{X_{t}\right\}_{t \in T}$ of smooth projective varieties parametrized by a smooth irreducible scheme $T / k$. 
By this we might mean that there is a $\widetilde{T}$ which is smooth over $W$ such that $\pi: \tilde{X} \rightarrow \widetilde{T}$ (is a projective morphism and) is a lifting of our family to $W$, and, moreover, that $R^{q} \pi_{*} \Omega_{X}^{p} / \tilde{T}$ is locally free over $\tilde{T}$ and commutes with base change for all $p, q$. A quite general (unpublished) theorem of Grothendieck says that the $m$-dimensional Newton polygon of $X_{t}$ rises under specialization of $t$.

Therefore, the "minimal position" of the Newton polygons of $X_{t}$ (in the Euclidean plane) is achieved by the generic member $X_{\eta}$ of the family.

We shall borrow (with precious little justification) a term used in connection with abelian varieties and say that $X_{0} / k$ is ordinary in dimension $m$ if the $m$-dimensional Newton polygon and the $m$-dimensional Hodge polygon of (a lifting of) $X_{0} / k$ agree.

As a result of the theorems cited so far,

Corollary. If $X_{t}$ is ordinary in dimension $m$ for some $t \in T$, then so is the general member $X_{\eta}$.

What is known about the existence of ordinary varieties?

THEOREM (LUBIN, MilLER [9]). The generic curve of genus $g$ in characteristic $p>0$ is ordinary (in dimension one, that being the only dimension in which a curve can fail to be ordinary).

Of course, in our formulation of the above theorem, we have appealed to the theorem of Deligne and Mumford assuring us of the existence of the generic curve of genus $g$.

TheOREM (MUMFORD [10]). Any "sufficiently general" abelian variety in characteristic $p$ is ordinary. (Again, dimension one is the only relevant dimension.)

Very little seems to be known about general hypersurfaces. It seems reasonable to hope that the general hypersurface of degree $d$ and dimension $N$ of characteristic $p$ is ordinary (the relevant dimension here is $N$ ).

Katz has shown (unpublished) that if $X$ is the general hypersurface of degree $d$ in $n$ homogeneous variables with $n \not \equiv 1 \bmod d$, then the Newton and Hodge polygons of $X$ have the same slope at the point $(0,0)$ in the Euclidean plane.

4. F-crystals and their associated spans. By an $\boldsymbol{F}$-crystal we shall mean a $W$-module $M$ of finite type, endowed with a Frobenius-linear endomorphism $\boldsymbol{F}$. We may 'linearize' this notion over $W$ be defining a new module $M^{(p)}=M \otimes_{W} W^{(p)}$ where $W^{(p)}$ is the module $W$, given a $W$-module structure by means of the Frobenius automorphism: $W \rightarrow W$. Then our $F$-crystal is a $W$-linear map, $F: M^{(p)} \rightarrow M$. 
Given an $\boldsymbol{F}$-crystal as above, there is an operation we can perform which destroys almost, but not quite, all of its structure. What remains will be of great interest to us, in our applications.

Definition. A span $\boldsymbol{F}: H \rightarrow M$ is a $W$-linear homomorphism of $W$ modules of finite type. Given an $\boldsymbol{F}$-crystal we may pass to its associated span by 'forgetting that $M^{(p)}$ bears any relation to $M$.'

Suppose we are given a span $F: H \rightarrow M$. Suppose further, as will be the case in our applications, that $H$ and $M$ are free over $W$ of the same rank, and $\boldsymbol{F}$ is an injection. What determines the above span up to isomorphism, in the category of all spans?

The classical 'invariant factor theorem' answers this question for us. It assures us that we may choose a $W$-basis of $M$ so that $F$ is representable by a diagonal matrix, whose diagonal entries are precisely

$$
1,1, \ldots, 1, p, p, \ldots, p, \quad p^{2}, \ldots, p^{2}, \ldots \quad \ldots, p^{m}, \ldots, p^{m} .
$$

Moreover, the quantities

$$
h^{j}=\text { the number of times } p^{j} \text { occurs on the diagonal }
$$

are invariants of the isomorphy class of the span, and conversely, these numbers completely determine the isomorphy class of the span.

DEFINITION. Let us refer to $h^{j}$ as the jth Hodge number of the span.

The above definition anticipates the following theorem:

THEOREM 2. Let $X / W$ be projective, smooth, and satisfy the hypothesis that $H^{q}\left(X, \Omega_{X / W}^{p}\right)$ are torsion-free. Consider the span associated to the $F$-crystal $F: H_{D R}^{m}(X / W) \rightarrow H_{D R}^{m}(X / W)$. The Hodge numbers of this span are equal to the corresponding Hodge numbers of $X / W$. Explicitly, $h^{j}=h^{j, m-j}$.

REMARK. Given an $\boldsymbol{F}$-crystal $\boldsymbol{F}: M^{(p)} \rightarrow M$ where $M$ is free of finite rank, and $\boldsymbol{F}$ is injective, we may define the Hodge polygon of this crystal to be the polygon, as defined in $\$ 2$, constructed using the Hodge numbers of its associated span.

One then has the very easy

LEMMA. The Newton polygon of an F-crystal lies above (or on) its Hodge polygon.

Consequently the Katz conjecture (Theorem 1) follows immediately from this lemma and Theorem 2.

SKETCH OF PROOF OF THE LEMMA. It is straightforward linear algebra. Begin by showing

(*) If the Hodge numbers $h^{i}$ of an $F$-crystal $M$ vanish for $i<c$, then the slopes of the Newton polygon of $M$ are greater than or equal to $c$. 
Then use assertion $\left(^{*}\right)$ for the $F$-crystals $\wedge^{t} M$ (tth exterior power) for all $t \geqq 1$ to get the desired system of inequalities.

The statement of the theorem above may be formulated without benefit of a lifting of $X_{0}$ to $W$ :

CONJECTURE FOR NONLIFTABLE VARIETIES. Suppose $X_{0} / k$ is proper and smooth. Suppose the Hodge spectral sequence of $X_{0} / k$ degenerates at $E_{1}$, and the crystalline cohomology groups $H_{\mathrm{cris}}^{*}\left(X_{0} / W\right)$ are free over $W$. Then the abstract Hodge numbers of its "m-dimensional span" are equal to corresponding Hodge numbers of $X_{0} / k$.

Explicitly,

$$
h^{j}=\operatorname{dim} H^{m-j}\left(X_{0}, \Omega_{X_{0}}^{j}\right)
$$

for all $j$, where $h^{j}$ is the jth abstract Hodge number of the span

$$
F: H_{\text {cris }}^{m}\left(X_{0} / W\right) \rightarrow H_{\text {cris }}^{m}\left(X_{0} / W\right) \text {. }
$$

I shall now present a sharpened version of Theorem 2. It is somewhat more technical, but it clarifies, I believe, some of the interrelations of the various structures which cohabit de Rham cohomology. Here I will assume that the reader is familiar with the two spectral sequences converging to de Rham cohomology $\bmod p$ :

$$
\begin{array}{ll}
\text { Hodge: } & E_{1}^{r, s}=H^{s}\left(X_{0}, \Omega^{r}\right) \Rightarrow H_{D R}^{r+s}\left(X_{0} / k\right), \\
\text { Conjugate: } & E_{2}^{r, s}=H^{r}\left(X_{0}^{\prime}, \Omega^{s}\right) \Rightarrow H_{D R}^{r+s}\left(X_{0} / k\right)
\end{array}
$$

(cf. [5]). Here $X_{0} / k$ is the closed fiber of $X / W$ and $X_{0}^{\prime}$ is defined to be the pullback under Frobenius:

$$
\begin{array}{ccc}
X_{0}^{\prime} & \rightarrow & X_{0} \\
\downarrow & & \downarrow \\
k & \rightarrow & k
\end{array}
$$

These spectral sequences both degenerate (at $E_{1}$ and $E_{2}$, respectively) under the hypothesis of Theorem 2.

THEOREM 3. Let $X / W$ satisfy the hypotheses of Theorem 2. Let $F: H \rightarrow M$ denote its associated m-dimensional span. Let $\bar{\nabla}$ denote reduction of a $W$-module $\bmod p$. Let $\bar{H}_{j} \subset \bar{H}$ for each $j \geqq 0$ denote the image in $\bar{H}$ of the submodule of $H, F^{-1}\left(p^{j} M\right)$.

Let $\bar{M}_{j} \subset \bar{M}$ for each $j \geqq 0$ denote the image in $\bar{M}$ of the submodule of $M$,

$$
\frac{1}{p^{j}} \boldsymbol{F}\left(\boldsymbol{F}^{-1}\left(p^{j} M\right)\right)
$$


Then under the identifications

$$
\bar{H} \cong H_{D R}^{m}\left(X_{0} / k\right), \quad \bar{M} \cong H_{D R}^{m}\left(X_{0} / k\right),
$$

the filtrations

$$
\begin{aligned}
& \{0\}=\bar{H}_{m+1} \subset \ldots \subset \bar{H}_{j} \subset \bar{H}_{j-1} \subset \ldots \subset \bar{H}_{0}=\bar{H}, \\
& \{0\} \subset \bar{M}_{0} \subset \ldots \subset \bar{M}_{m}=\bar{M}
\end{aligned}
$$

are identified with

$$
\text { the Hodge filtration on } H_{D R}^{m}\left(X_{0} / k\right) \text {, }
$$$$
\text { the conjugate filtration on } H_{D R}^{m}\left(X_{0} / k\right) \text {. }
$$

Moreover, after these identifications, there is a commutative diagram:

$$
\left.\underset{\frac{1}{p^{j}}}{F}\right|_{\frac{1}{p^{j}} \boldsymbol{F}\left(\boldsymbol{F}^{-1}\left(p^{j} M\right)\right) \rightarrow \bar{M}_{j} / \bar{M}_{j-1} \cong H^{j-j}\left(X_{0}^{\prime}, \Omega^{j}\right) .} ^{\left.H_{j}\right|_{j+1} \cong H^{m-j}\left(X_{0}^{\prime}, \Omega^{j}\right)}
$$

The general question. Removing the hypothesis of freeness of the $H^{p}\left(X, \Omega_{X / W}^{q}\right)$, it seems sensible to work with the entire complex $R \pi_{*} \Omega_{X / W}^{\cdot}$ in the derived category of $W$-modules where $\pi: X \rightarrow \operatorname{Spec} W$ is the structure map. This leads us to define the full de Rham span of $X / W$ as a morphism

$$
\boldsymbol{F}^{\bullet}: H^{\bullet} \rightarrow M^{\bullet}
$$

in the derived category. It would be tempting to hope that the de Rham span of $X / W$ can be reconstructed up to isomorphisms entirely by means of $\bmod p$ data-e.g., by means of the complex $R \pi_{0 *} \Omega_{X_{0} / k}^{\circ}$ endowed with its Hodge filtration, where the subscript 0 denotes reduction $\bmod p$.

5. Spans endowed with Hodge filtrations. Since Theorem 2 assures us that the spans associated to the de Rham cohomology groups of $X / W$ yield 'no new invariants of $X / W$ ' beyond the Hodge numbers, it is of interest to retain a bit more information concerning $X / W$ :

Definition. The $m$-dimensional filtered span of $X / W$ is the associated span of the $\boldsymbol{F}$-crystal,

$$
F: H_{D R}^{m}(X / W) \rightarrow H_{D R}^{m}(X / W)
$$


together with the Hodge filtration

$$
0 \subset H_{m} \subset H_{m-1} \subset \ldots \subset H_{0}=H_{D R}^{m}(X / W)
$$

on the domain.

The category of filtered spans is the category whose objects are spans $F: H \rightarrow M$ together with filtrations as above,

$$
0 \subset H_{m} \subset \ldots \subset H_{0}=H
$$

by sub- $W$-module direct summands.

Now there is a condition, which I will refer to as the divisibility property, which implies (whenever it holds) that even the filtered spans of $X / W$ yield no new invariants beyond the Hodge numbers.

LEMMA. Let

$$
0 \subset H_{m} \subset \ldots \subset H_{0}=H \stackrel{F}{\rightarrow} M
$$

be a filtered span such that $H$ and $M$ are free of the same rank, $\boldsymbol{F}$ is an injection, and

$$
h^{j}=\operatorname{rank}\left(H_{j} / H_{j+1}\right)
$$

where $h^{j}$ is the jth Hodge number of the span $F: H \rightarrow M$.

These are equivalent:

(1) $\boldsymbol{F}\left(H_{j}\right) \subset p^{j} M$ for all $j$.

(2) There is a W-basis of $H$, compatible with the filtration

$$
0 \subset H_{m} \subset \ldots \subset H_{0}=H
$$

(this means that $H_{j}$ is generated by the last $h^{j}+h^{j+1}+\ldots+h^{m}$ basis vectors) and a $W$-basis of $M$ such that the matrix of $\boldsymbol{F}$ with respect to these bases is a diagonal matrix, with diagonal entries

$$
1,1, \ldots, 1, p, p, \ldots \ldots, p^{m}, p^{m} .
$$

(3) Definition. The above filtered span is divisible.

I have no examples of smooth proper schemes $X / W$ whose filtered span is not divisible.

The following weak divisibility property is quite easy to establish:

$$
F H_{j} \subset p^{[j]} M
$$

where $p^{[j]}$ is the $j$ th divided power of the ideal generated by $p$. Explicitly, we may take

$$
[j]=\min _{n \geqq p} \operatorname{ord}_{p}\left(p^{n} / n !\right)
$$


Consequently, the divisibility property holds for the filtered spans of schemes $X / W$ of dimension less than $p=\operatorname{char}(k)$. It holds, therefore, for curves, and for abelian varieties.

In attempting to understand divisibility phenomena, I discovered some curious estimates [8]. For example, one may prove

$$
F H_{j} \subset \sum_{k<j} p^{[j-k]} F H_{j}+p^{j} M
$$

and, in particular,

$$
F H_{j} \subset p F H+p^{j} M .
$$

At the moment one lacks sufficient insight even to venture a guess as to whether the "divisibility property" holds generally and the sequence of curious estimates I obtain are a mere artifact of my method, or whether these estimates do reflect the intricacy of the phenomena.

It is interesting to consider the first unresolved case: $p=j$.

For this, let us concentrate on a $p$-dimensional scheme $X / W, p=\operatorname{char}(k)$, and take $m=p$.

The only obstruction to the divisibility property is given by the map

$$
\frac{1}{p^{p-1}} F: H_{p} \rightarrow M / \dot{p} M \text {. }
$$

Under the conditions of the theorem, one may re-express this obstruction as a bilinear map

$$
H^{0}\left(X_{0}, \Omega_{X_{0} / k}^{p}\right) \otimes H^{0}\left(X_{0}, \Omega_{X_{0} / k}^{p}\right) \stackrel{A}{\rightarrow} k .
$$

This bilinear form seems to be quite interesting. It depends upon the lifting $X / W$. It is alternating if $p=2$, and symmetric if $p \neq 2$.

Since it represents the (only) obstruction to the truth of the divisibility property for such $X / W$, the reader may have gathered that $\mathrm{I}$ have no examples where $A \neq 0$.

\section{REFERENCES}

1. J. Berthelot, A series of notes in the C. R. Acad. Sci. Paris Serr. A-B 269 (1969), A297-A300; A357-A360; A397-A400; ibid. 272 (1971), A141-A144; A1314-A1317; A1397-A1400. MR 40 \#151; \#2686; 41 \#8432.

2. B. Dwork, $A$ deformation theory for the zeta function of a hypersurface, Proc. Internat. Congress Math. (Stockholm, 1962), Inst. Mittag-Leffler, Djursholm, 1963, pp. 247-259. MR 31 \# 171 .

3. N. Katz, A note on a theorem of $A x$, Mimeographed Notes, Princeton University, Princeton, N.J., 1970.

4. Sci. Publ. Math. No. 35 (1968), 223-258. MR 39 \# 4168.

5. - Seminar on degeneration of algebraic varieties, Inst. for Advanced Study, Princeton, N.J., Mimeographed Notes, Fall Term, 1969/70. 
6. S. Kleiman, Weil cohomologies, Dix exposés sur la théorie des schémas, NorthHolland, Amsterdam.

7. Ju. I. Manin, The Hasse-Witt matrix of an algebraic curve, Izv. Akad. Nauk SSSR Ser. Mat. 25 (1961), 153-172; English transl., Amer. Math. Soc. Transl. (2) 45 (1965), 245-264. MR 23 \# A1638.

8. B. Mazur, Frobenius and the Hodge filtration (estimates), Ann. of Math. (to appear).

9. L. Miller, On curves with invertible Hasse-Witt matrix (to appear).

10. D. Mumford, Bi-extensions of formal groups, Internat. Colloq. Algebraic Geometry (Tata Inst. Fund. Res., Bombay, 1968), Oxford Univ. Press, London, 1969, pp. 307-322. MR 41 \# 1743.

11. E. Warning, Bemerkung zur vorstehenden Arbeit von Herr Chevalley, Abh. Math. Sem. Univ. Hamburg 11 (1936), 76-83.

12. A. Weil, Jacobi sums as "Grossencharaktere", Trans. Amer. Math. Soc. 73 (1952), 487-495. MR 14, 452. 